\title{
Tumor Boundary Extraction in Multislice MR Brain Images using Region and Contour Deformation
}

\author{
Albert K.W. Law, H. Zhu, F.K. Lam, Francis H.Y. Chan \\ Department of Electrical and Electronic Engineering, \\ The University of Hong Kong, Hong Kong \\ fhychan@eee.hku.hk
}

\author{
Brent C.B. Chan, P.P. Iu \\ Department of Radiology, \\ Kwong Wah Hospital, Hong Kong
}

\begin{abstract}
In this paper, we present a new approach for the extraction of brain tumor boundary in a series of $2 D M R$ image slices. The shape and position of tumor in one slice could be assumed to be similar to that in its neighboring slices. Using this correlation between consecutive images, the initial plan applied for each slice is extracted from the resulting boundary of the previous slice. The tumor boundary is located using region and contour deformation, which tolerates a rough initial plan. Therefore, only one coarse manual initial plan is required for the whole series of MR image slices. Performance of our approach is evaluated on MR image set. Comparisons with manual tracing show the accuracy and effectiveness of our approach.
\end{abstract}

Key words - deformable model, tumor boundary, MRI sequence.

\section{Introduction}

Managing non-surgical therapy of brain tumor involves periodic monitoring of tumor development in terms of area, volume, shape, etc. Routine magnetic resonance (MR) examination gives only a series of twodimensional (2D) image slices while subtle changes in the tumor may not be readily noticeable. Although radiologists can manually trace the tumor boundary in each of the 2D image slices for a rough estimation of its volume, it is really a tedious and time-consuming process. Routine application of this process would not be practical. Moreover, the results may not be consistent and repeatable owing to substantial intra-observer and inter-observer variability.

A series of parallel $2 \mathrm{D} M R$ images produce a $3 \mathrm{D}$ representation of the brain tumor. Successive $2 D$ MR images have some similarities between each other in terms of size, shape, axis, and gray intensity of the

0-7695-1113-9/01 \$10.00 ㄷ 2001 IEEE tumor. The change in various properties of tumor between one image and the next will be small when the slice thickness is kept within a certain value. In the analysis of successive $2 \mathrm{D} M R$ image slices, the traditional way was to process one by one separately. Active contour model [1] and various deformable models $[2,3]$ have been used to deal with this problem. However, the correlation between consecutive images was not taken into consideration. A range of methods [4-7] has been developed for the processing of medical image sequences. Most of them used geometric or shape constraints, which explicitly introduced a priori knowledge on the expected shape of contour. It is not suitable for this problem, as the shape of tumor can be different from case to case. Zhu and Yan [8] proposed an approach for the detection of brain tumor boundaries in a series of MR image slices using Hopfield neural network. The distance between actual boundary and initial boundary was limited by the search area. This complicated the problem and limited the range of applications.

In this paper, we present a new approach for the extraction of brain tumor boundary in a series of $2 \mathrm{D} M R$ image slices. For each of the slice, the initial plan applied is extracted from the resulting boundary of the previous slice. The boundary is located using region and contour deformation [9]. This method is suitable for this problem, as it can tolerate a rough initial plan. Then only one coarse manual initial plan is needed for the whole series of MR image slices. The tumor information between consecutive MR images is utilized. Performance of our approach is evaluated on MR image set.

\section{Methods}

The approach was to extract boundaries of brain tumors in a series of $2 \mathrm{D} \mathrm{MR}$ image slices, which is a sequence of parallel 2D images with known separation between each other. As the brain is a connected entity, it could be assumed that the shape and position of tumor in one slice should be similar to that in its neighboring 
slices. Based on this assumption, the detected boundary in the current slice can be used as initial plan for the next slice. Therefore, only one coarse initial plan is required at a convenient slice with an obvious tumor for the whole series of MR images. The major steps of our approach are shown in Figure 1. First, an initial slice is selected from the MR image set and an initial plan is set manually for tumor boundary detection. Then region and contour deformation is applied to locate tumor boundary. Finally, the tumor boundary is generated and it is also used as initial plan for the next slice.

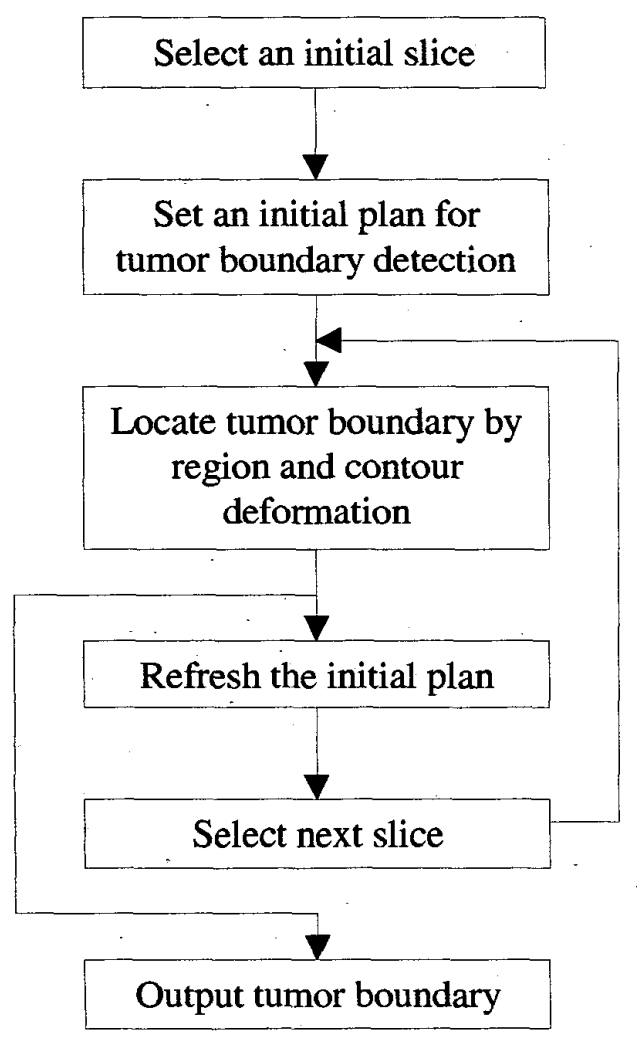

Figure 1. Block diagram of the brain tumor boundary extraction approach

The proposed approach uses a two step method [9], which performs region and contour deformation, to find the boundary of tumor from a fairly rough initial plan. The region and contour deformation are briefly summarized as below.

\subsection{Region deformation}

The region deformation [10] is to find a maximum area region with the same gray level distribution using a shrinking-growing method. The Kolmogorov-Smirnov (KS) test method is used to test whether the boundary pixel set and the object pixel set have the same gray level distribution. The Kolmogorov-Smirnov distance $D$ is defined as

$$
D=\max _{0 \leq g_{s} \leq 255}\left|F_{B}\left(g_{s}\right)-F_{o}\left(g_{s}\right)\right|
$$

where $F_{o}$ and $F_{B}$ are the gray level cumulative frequency distribution of object and boundary respectively. The hypothesis $F_{o}=F_{B}$ is accepted when $D<d$, and $d$, is defined as

$$
d=\frac{c}{\sqrt{\frac{A \cdot L}{(A+L)}}}
$$

where $c$ is the significance level of the test, $A$ is the area of object, and $L$ is the length of boundary.

When $D>d$, the region plan covers a region different from the tumor. Shrinking is performed to deform the region to meet $D<d$. Hence, the homogeneity of the region can be guaranteed. The shrinking algorithm is the erosion operation. It only deletes the region boundary elements, which has a different gray level distribution. When $D<d$, the region plan covers a region inside the tumor. The region, however, may not cover the whole tumor. To obtain the maximum area, growing is performed until $D>d$. The growing algorithm is the dilation operation. After each shrinking and growing, the new region area is compared with the previous one. If the region area does not change, the process stops; otherwise, the iteration of shrinking and growing continues.

\subsection{Contour deformation}

The boundary obtained from region deformation can be further refined by contour deformation [11]. The "snake" method [1] modeled the contour as an energyminimizing spline guided by internal and external forces.

$$
E_{\text {snake }}=\int_{0}^{1}\left[E_{\mathrm{int}}(v(s))+E_{\text {ext }}(v(s))\right] d s
$$

where $v(s)=(x(s), y(s))$ is the parametric equation of the contour, and $s$ is the arc length. 


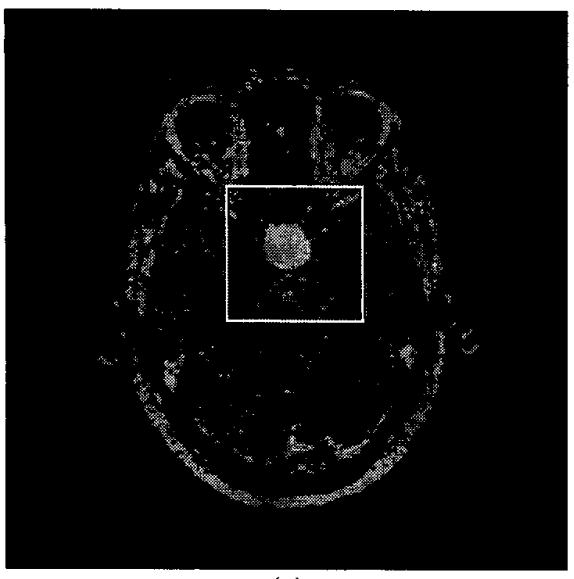

(a)

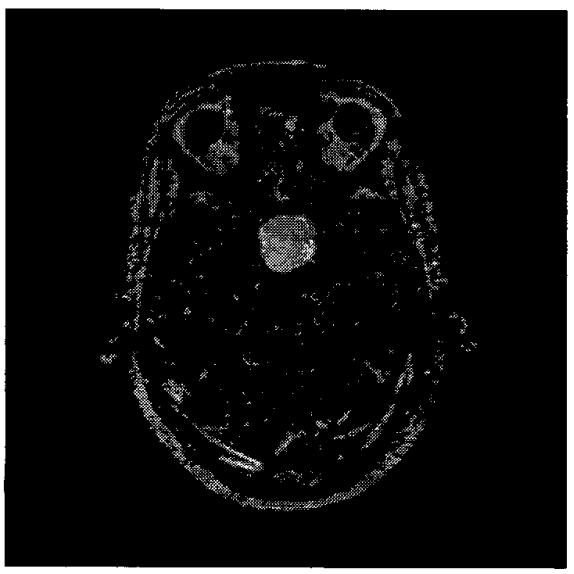

(b)

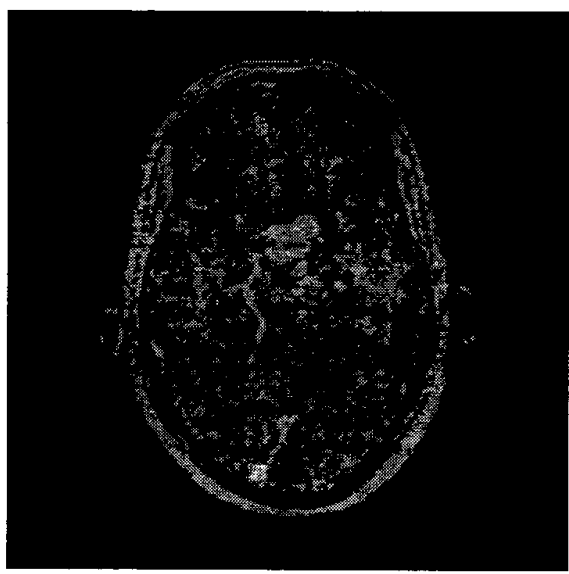

(c)

Figure 2. Selected image slices from the MR image set containing a brain tumor: (a) slice 35 , (b) slice 41 , (c) slice 49 .

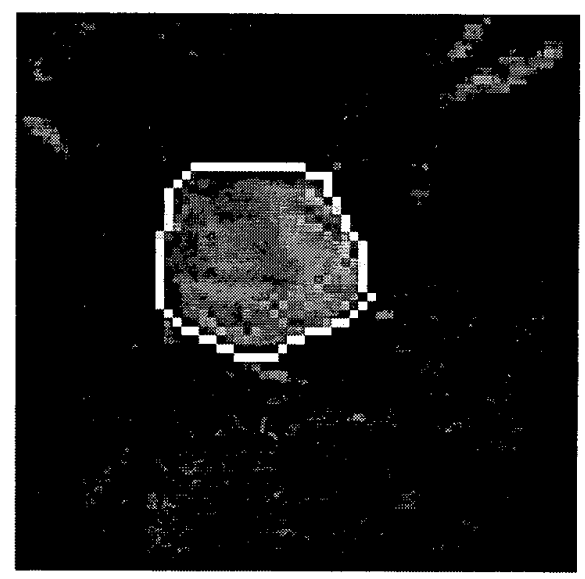

(a)

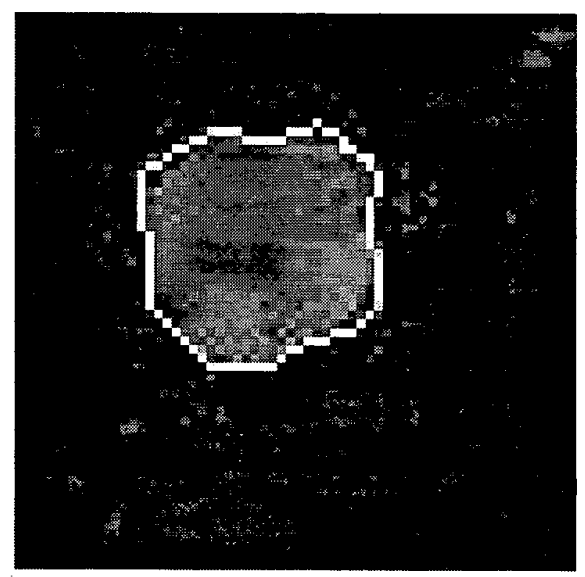

(b)

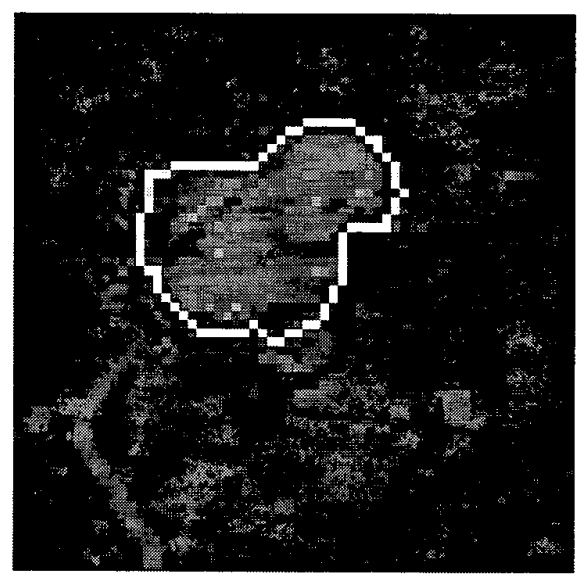

(c)

Figure 3. Extracted tumor boundaries, superimposed on the original image slices in Figure 2. Only a portion of the image [the rectangular region shown in Figure 2(a)] is shown here: (a) slice 35, (b) slice 41, (c) slice 49. 
The internal energy $E_{\text {int }}$ has two parts.

$$
E_{\mathrm{int}}=\left(\alpha(s)\left|v_{s}(s)\right|^{2}+\beta(s)\left|v_{s s}(s)\right|^{2}\right) / 2
$$

where $v_{s}$ and $v_{s s}$ are the first and second order derivatives, respectively. These two terms are used to control the continuity and smoothness of the contour, with $\alpha$ and $\beta$ representing the weights.

The external energy $E_{e x t}$ is from the image edge information. $\nabla$ is the gradient operator and $I$ is the image. $\nabla^{2} I$ is the second order derivatives of the image.

$$
E_{e x t}=\gamma(s) \log \left(1+\left|G_{\sigma} * \nabla^{2} I\right|\right)
$$

where $\not(s)$ is the weight and $G_{\sigma}$ is a Gaussian function of the standard deviation $\sigma$.

\subsection{Analysis of results from the approach}

Results from the proposed approach were compared with those from manual tracing by our radiologist (P.P.Iu). Double blind method was used in this analysis. One did not know the results from the other when performing one's own part. The result comparison was done afterwards for checking against each other. The percentage overlapped in area was determined for the results obtained from the proposed approach and manual tracing in each image. For the manual tracing, the Paint application from Windows Accessories was used. The image was enlarged to $200 \%$ and our radiologist used mouse to draw the tumor boundary with pencil tools in white color.

\section{Results}

Performance of the proposed approach is shown. A MR image set with 74 slices was used. Figure 2 shows several selected images from this set (8-bit grayscale, $256 \times 256$ pixels). The pixel size of each slice is $0.898 \mathrm{~mm} \times 0.898 \mathrm{~mm}$. The slice thickness is $0.8 \mathrm{~mm}$. According to our radiologist's analysis (P.P.Iu), slices 23-60 intersect the tumor. The middle tumor slices appeared relatively obvious and bigger among images of the whole tumor. The initial slice could be selected from one of those images with obvious tumor visible to user. Thus it is a better strategy to start from a middle tumor slice and process other slices in two directions: forward and backward. Here, slice 39 was selected as an example. After that, initial plan was set manually. The boundary located using region and contour deformation was used as initial plan for slices 38 and 40 . Our approach could work well for slices 28-50. Figure 3 shows the results of several selected slices from the MR image set. We found that the fast snake method [11] could also work well in the same slice range. To compare with manual tracing, the percentage overlapped in area was over $80 \%$ for slices $31-49$. The sampling rate of this image set was then reduced to simulate the increase in slice thickness. We increased the step length when selecting the next slice. To simulate the thickness of 3-5 mm, the step length should be 4-6 slices, corresponding to $3.2-4.8 \mathrm{~mm}$. Our approach could work well at any step length from 1 to 6 - slices. When step length increased to 4 slices, the fast snake method [1.1] failed. In the above experiments, the parameters $\alpha(s)$, $\beta(s)$, and $\mathcal{X}(s)$ were fixed at $1 ; 1$, and 10 respectively. The values of two other parameters, $c$ and $\sigma$, were determined experimentally.

\section{Discussion and Conclusions}

In our approach, no assumption was made on the gray level distribution of tumor. There was no restriction on the position of initial plan. so it could be far away from the actual boundary of brain tumor. The tumor boundary in each slice was extracted with pixel accuracy in the MR image set. The results of the proposed approach were close to that of manual tracing for the middle part of the tumor. It is reasonable, as the middle part is bigger and clearer than other parts of the tumor. Our approach could work well at larger step length than the fast snake method [11]. Thus, it is robust for the brain tumor boundary extraction in MR image set. This helps doctors to monitor different properties of brain tumor at each stage, as it is very difficult to detect minor tumor changes by viewing the MR images. The detected tumor boundary in each slice can also be used for 3D rendering and volume measurement.

\section{Acknowledgment}

The authors would like to thank Image Processing Group at Guy's Hospital, London, U.K. for providing the MR image set. This work was supported in part by Hong Kong Research Grants Council under Grants HKU553/96M and HKU7036/97E.

\section{References}

[1] M. Kass, A. Witkin. D. Terzopoulos, "Snakes, active contour models", Proc. First Int. Conf. Comput. Vision, 1987, pp. 259-268.

[2] A.K. Jain, Y. Zhong, M.-P. Dubuisson-Jolly, "Deformable template models: A review", Signal Process., 1998, vol. 71, pp. 109-129.

[3] T. McInerney, D. Terzopoulos, "Deformable models in medical image analysis: a survey", Medical Image Analysis, 1996, vol. 1, pp. 91-108. 
[4] Y. Nakazawa, T. Saito, "Region extraction with standard brain stlas for analysis of MRI brain images", IEEE Int. Conf. Image Process., 1994, vol. 1, pp. 387-391.

[5] F. Howing, D. Wermser, L.S. Dooley, "Recognition and tracking of articulatory organs in X-ray image sequences", Electron. Lett., 1996, vol. 32, pp. 444-445.

[6] D.J. Kang, C.Y. Kim, Y.S. Seo, L.S. Kweon, "Fast and stable method for detecting and tracking medical organs in MRI sequences", IEICE Trans. Inf. Syst., 1999, vol. 82, pp. 497-499.

[7] D.J. Kang, "Stable snake algorithm for convex tracking of MRI sequences", Electron. Lett., 1999, vol. 35, pp. 10701071.
[8] Y. Zhu, H. Yan, "Computerized tumor boundary detection using a Hopfield neural network", IEEE Trans. Med. Imaging, 1997, vol. 16, pp. 55-67.

[9] F.H.Y. Chan, F.K. Lam, P.W.F. Poon, H. Zhu, K.H. Chan, "Object boundary location by region and contour deformation", IEE Proc. Vis. Image Signal Process., 1996, vol. 143, pp. 353-360.

[10] H. Zhu, F.H.Y. Chan, F.K. Lam, P.W.F. Poon, "A deformable region model for locating the boundary of brain tumor", Proc.17th Ann. Conf. IEEE EMBS, 1995, vol. 1, pp. 411-412.

[11] D.J. Williams, M. Shah, "A fast algorithm for active contours and curvature estimation", CVGIP: Image Underst., 1992, vol. 55, pp. 14-26. 